\title{
Entropy production and wave packet dynamics in the Fock space of closed chaotic many-body systems.
}

\author{
V.V. Flambaum ${ }^{1}{ }^{*}$ and F.M. Izrailev ${ }^{2}$ \\ ${ }^{1}$ School of Physics, University of New South Wales, Sydney 2052, Australia \\ ${ }^{2}$ Instituto de Física, Universidad Autónoma de Puebla, Apartado Postal J-48, Puebla 72570, México
}

\begin{abstract}
Highly excited many-particle states in quantum systems such as nuclei, atoms, quantum dots, spin systems, quantum computers etc., can be considered as "chaotic" superpositions of mean-field basis states (Slater determinants, products of spin or qubit states). This is due to a very high level density of many-body states that are easily mixed by a residual interaction between particles (quasiparticles). For such systems, we have derived simple analytical expressions for the time dependence of energy width of wave packets, as well as for the entropy, number of principal basis components and inverse participation ratio, and tested them in numerical experiments. It is shown that the energy width $\Delta(t)$ increases linearly and very quickly saturates. The entropy of a system increases quadratically, $S(t) \sim t^{2}$ at small times, and after, can grow linearly, $S(t) \sim t$, before the saturation. Correspondingly, the number of principal components determined by the entropy, $N_{p c} \sim \exp (S(t))$, or by the inverse participation ratio, increases exponentially fast before the saturation. These results are explained in terms of a cascade model which describes the flow of excitation in the Fock space of basis components. Finally, a striking phenomenon of damped oscillations in the Fock space at the transition to an equilibrium is discussed.
\end{abstract}

PACS numbers: 03.67.Lx, 05.45.Mt, 24.10.Cn

\section{INTRODUCTION}

Highly excited many-particle states in many-body systems quite often can be presented as "chaotic" superpositions of shell-model basis states, see recent calculations for complex atoms [1], multicharged ions [2], nuclei [3] and spin systems [4, [5. The origin of this phenomenon relates to a very high density of many-particle energy levels, which increases drastically with an increase of energy. Indeed, the number $N$ of combinations in the distribution of $n$ particles (or quasi-particles) over $m$ "orbitals" (single-particle states) is exponentially large $(N \sim m ! / n !(m-n) !$ in a Fermi system). Therefore, the spacing $D$ between many-body levels is exponentially small and a "residual" interaction $V$ between the particles can mix a huge number of the basis states of a mean field $H_{0}$ (Slater determinants), when forming exact eigenstates of the total Hamiltonian $H=H_{0}+V$.

The onset of chaos for highly excited states, as well as for many-particle spectra, has been recently studied in great detail in terms of the Two-Body Random Interaction (TBRI) model which was invented about three decades ago [6]. In this model all two-body matrix elements are assumed to be independent and random variables, therefore all dynamical correlations are neglected. Thus, the TBRI model is essentially the random matrix model, however, it differs from standard Random Matrix Models where the two-body nature of interaction is not taken into account (see, e.g. [7] 9] and review [10]).
One of the important results obtained recently [11] in the frame of this model is the Anderson-like transition which occurs in the Fock space determined by manyparticle states of $H_{0}$. The critical value $V_{c r}$ for this transition is determined by the density of states $\rho_{f}=d_{f}^{-1}$ of those basis states which are directly coupled by a twobody interaction [7,8,11, 13]. When the interaction is very weak, $V_{0} \ll d_{f}$, exact eigenstates are delta-like functions in the unperturbed basis, with a very small admixture of other components which can be found by the standard perturbation theory. With an increase of the interaction, the number of principal components $N_{p c}$ increases and can be very large, $N_{p c} \gg 1$. However, if the interaction is still not too strong, $\pi^{-2} \sqrt{d_{f} D} \ll V_{0} \leq d_{f}$ [8], the eigenstates are sparse, with extremely large fluctuations of components. In order to have ergodic eigenstates which fill some energy range (see below), one needs to have the perturbation large enough, $V_{0} \gg d_{f}$ (for a large number of particles this transition is sharp and, in fact, one needs the weaker condition, $V_{0} \geq d_{f}$ ).

Above the threshold of chaos, $V_{0} \geq d_{f}$, the number of principal basis components in an eigenstate can be estimated as $N_{p c} \sim \Gamma / D$ where $\Gamma$ is the spreading width of the strength function. In such chaotic eigenstates any external weak perturbation is exponentially enhanced. The enhancement factor can be estimated as $\sqrt{N_{p c}} \propto 1 / \sqrt{D}$, see e.g. 114 and references therein. This huge enhancement have been observed in numerous experiments when

*email address: flambaum@newt.phys.unsw.edu.au 
studying parity violation effects in compound nuclei (see, for example, review [15]).

In recent works 16,17] the theory of many-body chaos has been extended to quantum computers. Since in this case the density of energy levels is extremely high, it is often impossible to resolve particular many-body levels. This happens for the injection of an electron into a many-electron quantum dot, for the capture of an energetic particle by a nucleus or atom, or for different models of quantum computer with a large number of interacting qubits (spins). In this case the approach based on the study of stationary chaotic eigenstates turns out to be not an adequate one, and one should consider the time evolution of a wave function and entropy [17].

In contrast to the study of spectra and eigenstates, the analysis of the evolution of wave packets in random matrix models is based mainly on numerical results. In the first line, one should mention the numerical study [18] of Band Random Matrices that describe quasi-1D disordered models with finite number of channels. Recently, the attention has been paid to the so-called Wigner Band Random (WBRM) model [19] which is used in the study of generic properties of strength functions in dependence on the strength of interaction 2021. In particular, in Refs. 22,23 the problem of the quantum-classical correspondence for the time evolution of wave packets was under close study. We note that the WBRM model serves as convenient random matrix model for different quantum systems, and in many aspects can be compared with the TBRI model [24].

In this paper we study generic properties of the evolution of wave packets in the energy shell, paying the main attention to the time-dependence of the entropy, width of packets, and inverse participation ratio. We derive analytical estimates for these quantities and check numerically our predictions with the use of the TBRI and WBRM models.

\section{TIME EVOLUTION OF CHAOTIC MANY-BODY STATES}

Exact ("compound") eigenstates $|k\rangle$ of a many-body Hamiltonian $H=H_{0}+V$ can be expressed in terms of simple shell-model basis states $|f\rangle$ (eigenstates of $H_{0}$ ), or by products of qubits in a model of quantum computer:

$$
\left.|k\rangle=\sum_{f} C_{f}^{(k)}|f\rangle ; \quad|f\rangle=\left.a_{f_{1}}^{+} \ldots a_{f_{n}}^{+}\right|^{\prime \prime} \text { vacuum }^{\prime \prime}\right\rangle .
$$

Here $a_{s}^{+}$are creation, or spin-raising, operators (in the latter case, the ground state $\mid{ }^{\prime \prime}$ vacuum $\left.{ }^{\prime \prime}\right\rangle$ corresponds to the situation with all spins down), and $C_{f}^{(k)}$ are components of compound eigenstates $|k\rangle$ formed by the residual interaction $V$.
In what follows, we consider the time evolution of the system for the case when compound eigenstates are chaotic. By this term we mean that the number of principal components is very large, $\sqrt{N_{p c}} \gg 1$, and the components $C_{f}^{(k)}$ can be treated as uncorrelated amplitudes with the gaussian distribution around their mean values, see details in [8]. Let us assume that initially $(t=0)$ the system is in a specific basis state $|0\rangle$ (with certain orbitals occupied, or, in the case of a quantum computer, when the state with certain spins "up" is prepared). This state can be presented as a sum over exact eigenstates of the total Hamiltonian $H$ :

$$
|0\rangle=\sum_{k} C_{0}^{(k)}|k\rangle .
$$

Then the time-dependent wave function reads as

$$
\Psi(t)=\sum_{k, f} C_{0}^{(k)} C_{f}^{(k)}|f\rangle \exp \left(-i E^{(k)} t\right) .
$$

Here the sum is taken over the compound states $k$ and basis states $f$, and we set $\hbar=1$.

The probability $W_{0}=\left|A_{0}\right|^{2}=|\langle 0 \mid \Psi(t)\rangle|^{2}$ to find the system in the initial state is determined by the amplitude

$$
\begin{array}{r}
A_{0}=\langle 0|\exp (-i H t)| 0\rangle=\sum_{k}\left|C_{0}^{(k)}\right|^{2} \exp \left(-i E^{(k)} t\right) \simeq \\
\int d E P_{0}(E) \exp (-i E t) .
\end{array}
$$

Here we replaced the summation over a large number of the eigenstates by the integration over their energies $E \equiv E^{(k)}$, and introduced the strength function (SF) $P_{0}(E)$ which is also known in the literature as the local spectral density of states, LDOS,

$$
P_{0}(E) \equiv \overline{\left|C_{0}^{(k)}\right|^{2}} \rho(E),
$$

with $\rho(E)$ as the density of exact eigenstates.

As one can see, the probability $W_{0}$ is entirely determined by the strength function (5). It is well known that in many applications this function has the Breit-Wigner (BW) form with a half-width $\Gamma_{0}=2 \pi \rho H_{f}^{2}$. In our case $\rho=\rho_{f}$ is the density of directly coupled basis states and $H_{f}^{2}=\overline{\left|H_{0 f}\right|^{2}}$ is the variance of the non-zero off-diagonal elements of $H$, defined by the residual interaction $V$. One should remind that in real situations the second moment $\Delta_{E}^{2}$ of the $\mathrm{SF}$ is always finite due to a finite range of interaction in the energy representation. Therefore, the Breit-Wigner form of the SF can occur for a finite energy range only, determined by the energy width of the interaction.

However, it was recently shown (see e.g. [8,13] and references therein) that if $\Gamma_{0}$ defined by the above expression, is of the order (or larger) of the mean-square-root width $\Delta_{E}$ of the SF itself, the form of the SF in the TBRI model is very close to the Gaussian. Strong deviations 
of the SF from the BW-dependence have been observed numerically when studying the structure of the SF and eigenfunctions of the Ce atom [1]. Also, numerical data [3] have revealed that the form of the SF in nuclear shell models is much closer to the Gaussian rather that to the BW. This results from the fact that the three orbitals $s$, $d_{3 / 2}$ and $d_{5 / 2}$ included into the nuclear shell-model calculation [3], have close mean-field energies and the residual interaction $V$ plays the dominant role in the Hamiltonian matrix.

Recent analytical results 13 for the TBRI model allow to describe the whole transition for the SF from the BW regime to that of the Gaussian. This model is characterized by two-body random matrix elements which determine the residual interaction $V$ between $n$ Fermiparticles occupying $m$ orbitals (single-particle states), see details and references, for example, in 8,24. It was shown that in general case the $\mathrm{SF}$ is given by the following approximate expression [25, 13,

$$
\begin{gathered}
P_{0}(E)=\frac{1}{2 \pi} \frac{\Gamma(E)}{\left(E_{0}+\delta_{0}(E)-E\right)^{2}+\Gamma^{2}(E) / 4}, \\
\Gamma(E) \simeq 2 \pi \overline{\left|H_{0 f}\right|^{2}} \rho_{f}(E) .
\end{gathered}
$$

One can see that the general expression (6) is of the BreitWigner form, however, with $\Gamma(E)$ as some function of the total energy $E$. Here $\delta_{0}(E)$ is the correction to the unperturbed energy level $E_{0}$ due to the residual interaction $V$, and $\rho_{f}(E)$ is the density of those basis states $|f\rangle$ which are directly coupled by the interaction $H_{0 f}$ with the initial state $|0\rangle$. It was shown [13] that for the TBRI model the function $\Gamma(E)$ has the Gaussian form with the variance which depends on the model parameters. In the case of relatively small (but non-perturbative) interaction, $\Gamma_{0}=2 \pi \rho_{f} H_{f}^{2} \ll \Delta_{E}$, the function $\Gamma(E)$ is very broad (i.e. it does not change significantly within the energy intervals $\sim \Gamma$ and $\Delta_{E}$ ) and can be treated as the constant, $\Gamma(E) \simeq \Gamma_{0}$. In the other limit case of a strong interaction, $\Gamma_{0} \geq \Delta_{E}$, the dependence $\Gamma(E)$ in (6) is the leading one, and the slow dependence in the denominator can be neglected. One should note that the simple expression (7) for the width $\Gamma(E)$ has to be modified in this limit, see details in 13 .

The second moment $\Delta_{E}^{2}$ of the SF can be found from the equation $\Delta_{E}^{2}=\sum_{f \neq 0} H_{0 f}^{2}$. Here the summation is taken over the off-diagonal matrix elements $H_{0 f}$ which couple the initial state $|0\rangle$ with the others $|f\rangle$. For the TBRI model the analytical expression for $\Delta_{E}$ has been obtained in 8 ,

$$
\Delta_{E}^{2}=\frac{1}{4} V_{0}^{2} n(n-1)(m-n)(m-n+3)
$$

where $V_{0}^{2}$ is the variance of the off-diagonal matrix elements of the two-body residual interaction $V$. In fact, for
Fermi-particles the width $\Delta_{E}$ turns out to be the same for any basis state $|0\rangle$.

Let us first start with the probability $W_{0}(t)$ of the system to stay in the initial state. In two limit cases of small and very large times, the dependence $W_{0}$ is shown [17,26. to be of the following forms,

$$
W_{0}(t)=\exp \left(-\Delta_{E}^{2} t^{2}\right) ; \quad t \ll \frac{\Gamma_{p}}{\Delta_{E}^{2}}
$$

and

$$
W_{0}(t)=C \exp \left(-\Gamma_{p} t\right) ; \quad t \gg \frac{\Gamma_{p}}{\Delta_{E}^{2}} .
$$

Here $\Gamma_{p}$ is the imaginary part of the pole of the SF (see Eq.(6) in the complex energy plane. In the case when the SF has the standard BW form, we have the obvious relation $\Gamma_{p}=\Gamma_{0}$ where the latter is given by the Fermi golden rule. In other limit of a strong interaction, when the SF has the Gaussian form, the expression for $\Gamma_{p}$ is not simple. The transition from one regime of the time dependence of $W_{0}(t)$ to another is schematically shown in Fig.1.

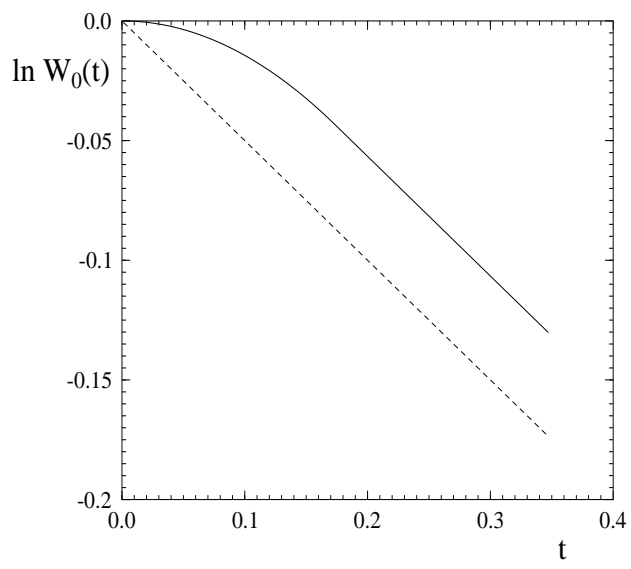

FIG. 1. Schematic time dependence of $W_{0}(t)$ for $\Gamma_{p}=0.5, \Delta_{E}=1.2$; the point $t_{c}$ where the dependence $(9)$ is changed to 10 is $t_{c}=\Gamma_{p} / \Delta_{E}^{2} \approx 0.17$.

Now we estimate the probabilities $w_{f}=\left|A_{f}(t)\right|^{2}$ to find the system in other basis states. For a very small time we have,

$$
w_{f}=\left|\left\langle f\left|e^{-i H t}\right| 0\right\rangle\right|^{2} \simeq\left|H_{0 f}\right|^{2} t^{2} .
$$

Note, that only the states directly connected to the initial state are populated at this time scale. One can estimate the population of these states for a larger time by substituting the time-dependent wave function $\Psi(t)=$ $A_{0}(t) \psi_{0}+\sum_{f} A_{f}(t) \psi_{f}$ into the Schrodinger equation,

$$
i \hbar \frac{d A_{f}}{d t}=H_{0 f}(t) A_{0}+\sum_{k} H_{f k}(t) A_{k} .
$$


Here $k, f \neq 0$ and $H_{0 f}(t) \equiv H_{0 f} \exp \left(i \omega_{0 f} t\right)$. Note that the second term in the right-hand-side may be treated as a random variable with the zero mean value. Indeed, $A_{k} \propto H_{0 k}$, therefore, $\overline{H_{f k}(t) H_{0 f}}=0$. The variance of this term is $\overline{\sum_{k}\left|H_{f k}(t) A_{k}\right|^{2}}=\overline{\left|H_{f k}(t)\right|^{2}} \sum_{k}\left|A_{k}\right|^{2}=$ $\overline{\left|H_{f k}(t)\right|^{2}}\left(1-\left|A_{0}\right|^{2}\right)$. Comparing this with the first term in the right-hand-side of Eq.(12), one may conclude that the second term is not very important for small times when $A_{0}(t) \sim 1$. Neglecting the second term and assuming $\left|A_{0}(t)\right|=\exp (-\Gamma t / 2)$ which is valid for $\Gamma<<\Delta_{E}$, we obtain 17,

$$
\begin{array}{r}
w_{f}=\left|H_{0 f}\right|^{2}\left|\int_{0}^{t}\right| A_{0}(t)\left|e^{i \omega_{0 f} t} d t\right|^{2} \simeq \\
\frac{\left|H_{0 f}\right|^{2}}{\omega_{0 f}^{2}+\Gamma^{2} / 4}\left|e^{\left(i \omega_{0 f}-\Gamma / 2\right) t}-1\right|^{2},
\end{array}
$$

where $\omega_{0 f}=E_{f}-E_{0}$. This approximate estimate shows that only the basis states within the energy interval $\Gamma$ can be substantially populated (if $\Gamma>\Delta_{E}$, this energy interval is equal to $\Delta_{E}$ ).

For large time, the result is different for perturbative and chaotic regimes. In the perturbative regime the expression (13) for $w_{f}$ is the final one. In the chaotic regime the asymptotic expression for $t \rightarrow \infty$ can be obtained in the following way. The projection of $\Psi(t)$ (see Eq. (3)) onto the state $f$ gives

$$
\begin{gathered}
w_{f}(t)=w_{f}^{s}+w_{f}^{\text {fluct }}(t), \\
w_{f}^{s}=\sum_{k}\left|C_{0}^{(k)}\right|^{2}\left|C_{f}^{(k)}\right|^{2} \simeq \int \frac{d E}{\rho(E)} P_{0}(E) P_{f}(E) \simeq \\
\frac{1}{2 \pi \rho} \frac{\Gamma_{t}}{\left(E_{0}-E_{f}\right)^{2}+\left(\Gamma_{t} / 2\right)^{2}} .
\end{gathered}
$$

Here the result is written for the case when the SF has the BW form both for initial $|0\rangle$ and final state $|f\rangle$ with the corresponding half-widths $\Gamma_{0}^{0}$ and $\Gamma_{0}^{f}$. In this case the resulting form of $w_{f}^{s}$ is, approximately, again the BreitWigner with the new half-width $\Gamma_{t} \simeq \Gamma_{0}^{0}+\Gamma_{0}^{f} \simeq 2 \Gamma_{0}$. However, if $\Gamma_{0} \geq \Delta_{E}$, the form of $w_{f}^{s}$ is close to the Gaussian with the variance $\left(\Delta_{E}\right)_{t}^{2} \simeq 2 \Delta_{E}^{2}$ 17.

The term $w_{f}^{\text {fluct }}(t)$ can be written in the form,

$$
w_{f}^{f l u c t}(t)=\sum_{k, p ; k \neq p} C_{0}^{(k)} C_{f}^{(k)} C_{0}^{(p)} C_{f}^{(p)} \exp \left(i\left(E^{(k)}-E^{(p)}\right) t\right) .
$$

At large time, $t \rightarrow \infty$, the terms in the sum rapidly oscillate and one can put $\overline{w_{f}^{\text {fluct }}(t)}=0$. Thus, asymptotically the distribution of the components in the time-dependent wave function is close to that given by the form of the strength function (see Eqs.(5, 6) ), with a slightly larger spreading width.

Note that similar expression for $W_{0}$ contains the term $\left|C_{0}^{(k)}\right|^{4}$. For gaussian fluctuations of the components $C_{0}^{(k)}$, one can get $\overline{\left|C_{0}^{(k)}\right|^{4}}=3\left(\overline{\left|C_{0}^{(k)}\right|^{2}}\right)^{2}$ which is the known result in the Random Matrix Theory [29]. Therefore, if the number of principal components $N_{p c}$ in the SF is very large, the probability to find the system in the initial state $|0\rangle$ at large time is, at least, three times larger than the probability to find the system in any other state $|f\rangle$, see Figs. 2,3.

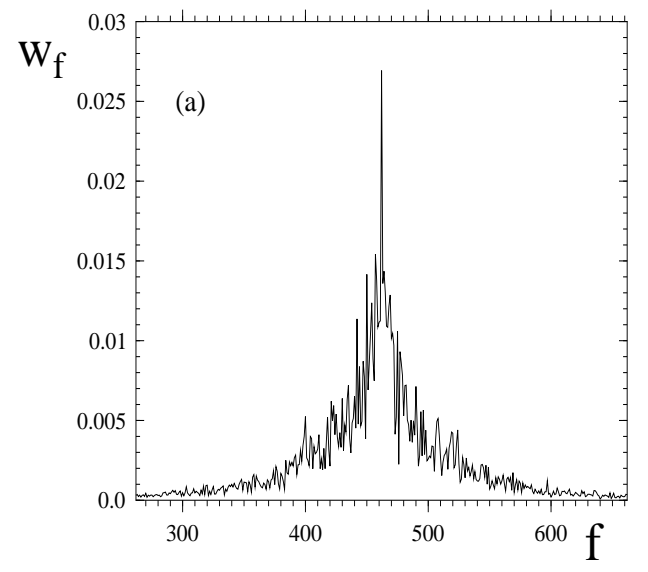

FIG. 2. Asymptotic distribution $w_{f}$ for the case when the strength function is of the Breit-Wigner form in the TBRI model. The parameters are: $n=6, m=12, V_{0}^{2} \approx 0.003$, $\Gamma_{0} \approx 0.50, \Delta_{E} \approx 1.16$, with the average over $N_{g}=10$ matrices with different realization of random two-body matrix elements, see in the text.

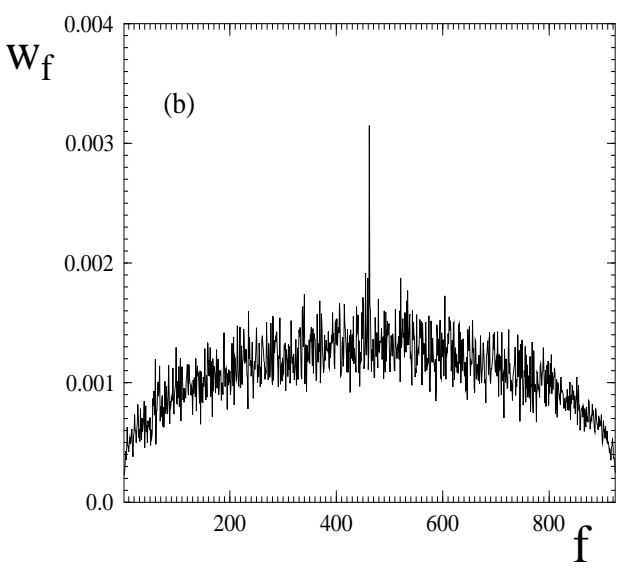

FIG. 3. Asymptotic distribution $w_{f}$ for the case when the strength function is close to the Gaussian. The only difference from Fig. 2 is the interaction strength, $V_{0}^{2} \approx 0.083$, correspondingly, $\Gamma_{0} \approx 10.5$ and $\Delta_{E} \approx 5.8$; the average was taken over $N_{g}=50$ matrices. 
In these figures the distribution of probabilities $w_{f}$ in the TBRI model is shown after a very long time $t=40$ for two different strengths of interaction (in fact, the time $t$ is measured in units $\hbar / d_{0}$ where $d_{0}=<\epsilon_{s+1}-\epsilon_{s}>$ is the mean level spacing between single-particle energies $\epsilon_{s}$ ). In both cases $n=6$ Fermi-particles occupy $m=12$ orbitals, therefore, the total number of many-particle states (the size of the Hamiltonian matrix) is $N=924$. The distribution of $\epsilon_{s}$ is taken random with $d_{0}=1$. Twobody matrix elements are taken as gaussian random entries with the zero mean and variance $V_{0}^{2}$, and in order to reduce the fluctuations, the distribution $w_{f}$ is obtained with an average over a number $N_{g}$ of matrices with different random realizations. Initially, only one basis state $n_{0}=462$ was populated at the center of the energy spectrum, in order to avoid the asymmetry of the distribution in the basis representation.

The two different values of $V_{0}$, for which the distributions $w_{f}$ are obtained, are chosen in such a way that in one case, see Fig.2, the strength function has the BreitWigner form, and in the other, the form is very close to the Gaussian (Fig.3). We should remind that the above two forms occur in the energy representation, however, the results are shown in the basis representation. These two representations are related through the density of states which is known to be of the Gaussian form for large number of particles and orbitals [6,29.

One can see that in both cases, Fig.2-3, the probability to stay in the original basis state is much larger than in the nearest ones. Comparing with the result of the standard random matrix theory, one can say that there is a noticeable difference (namely, the enhancement factor in Fig.3 is about 2.3, instead of 3.0).

\section{THE CASCADE MODEL}

One of the important question is how the entropy of quantum isolated systems increases in time at the transition to equilibrium. It is natural to define the entropy of a many-body state through the Shannon entropy,

$$
S(t)=-\sum_{k} w_{k} \ln w_{k}=-W_{0} \ln W_{0}-\sum_{f \neq 0} w_{f} \ln w_{f} .
$$

Here $W_{0}(t)=\left|A_{0}(t)\right|^{2}$ is the probability for the system to be in the initial state, and $w_{f}(t)=\left|A_{f}(t)\right|^{2}$ is the probability to be in the basis state $|f\rangle$. In what follows we assume that the initial conditions are $W_{0}(0)=1$ and $w_{f}(0)=0$, therefore, the entropy is equal to zero for $t=0$.

In order to study the evolution of a many-particle system with two-body interaction, it is convenient to introduce sub-classes for all basis states in the following way. The first class contains those $N_{1}$ basis states which are directly coupled with an initial state by the two-body interaction given by matrix elements $H_{0 f}$ of the interaction. Correspondingly, the second class consists of $\mathrm{N}_{2}$ basis states which are coupled with the initial one in the second order of the perturbation, this coupling is determined by $H_{0 \alpha} H_{\alpha f}$, etc.

Let us first consider the evolution for a large time $t \gg \frac{\Gamma}{(\Delta E)^{2}}$ (below we assume the BW shape of the SF). For this case the probabilities of the states in different classes can be determined by the "probability conservation equations",

$$
\begin{gathered}
\frac{d W_{0}}{d t}=-\Gamma W_{0} \\
\frac{d W_{1}}{d t}=\Gamma W_{0}-\Gamma W_{1} \\
\ldots \ldots \ldots \\
\frac{d W_{k}}{d t}=\Gamma W_{k-1}-\Gamma W_{k} \\
\ldots \ldots
\end{gathered}
$$

Here $W_{k}$ is the probability for the systems to be in the class $k$. The first term $\Gamma W_{k-1}$ in the right-hand-side of (18) is responsible for the flux from the previous class, and the second term $\Gamma W_{k}$ describes the decay of the states in the class $k$, into the next class $k+1$. We assume that the probability of the return to the previous class can be neglected. This is a valid approximation if the number of states $N_{k+1}$ in the next class is large in comparison with $N_{k}$ of the previous class. This approach can be compared with those based on the Caley tree model [11 where the flow from each state goes into other $M$ states, therefore, $N_{k} \simeq M^{k}$ with $M \gg 1$. Note that here we consider a system which is far from the equilibrium. Indeed, if the system is in the equilibrium, the probabilities of all states within the energy shell defined by the relation $\left|E_{f}-E_{0}\right| \leq \Gamma$, are of the same order, $w_{f} \simeq N_{p c}^{-1}$, with $N_{p c}$ as the total number of states inside the energy shell. Therefore, in order to neglect the return flux, one needs the condition $w_{f}=W_{k} / N_{k} \gg 1 / N_{p c}$ to be fulfilled.

Equations (18) have the simple solution,

$$
\begin{gathered}
W_{0}=\exp (-\Gamma t) \\
W_{n}=\frac{(\Gamma t)^{n}}{n !} \exp (-\Gamma t)=\frac{(\Gamma t)^{n}}{n !} W_{0} .
\end{gathered}
$$

The maximal probability $W_{n}=\frac{n^{n}}{n !} \exp (-n) \approx 1 / \sqrt{2 \pi n}$ to be in the class $n$ determined by the condition $\frac{d W_{n}}{d t}=0$ , occurs for $t=n / \Gamma$, therefore, this solution (19) can be considered as a cascade in the population of different classes . Indeed, at small times $t \ll \tau \equiv 1 / \Gamma$ the system is practically in the initial state, at times $t \approx \tau$ the flow 
spreads into the first class, for $t=n \tau$ it spreads into the $n$-th class, etc. For an infinite chain one can easily check the normalization condition,

$$
\sum_{n=0}^{\infty} W_{n}=\exp (-\Gamma t) \sum_{n=0}^{\infty} \frac{(\Gamma t)^{n}}{n !}=1 .
$$

\section{TIME DEPENDENCE OF THE ENTROPY}

The above expressions allow us to find the timedependence of the entropy,

$$
\begin{array}{r}
S(t) \approx-\sum_{n=0}^{\infty} W_{n} \ln \frac{W_{n}}{N_{n}}= \\
\Gamma t \ln M+\Gamma t-e^{-\Gamma t} \sum_{n=0}^{\infty} \frac{(\Gamma t)^{n}}{n !} \ln \frac{(\Gamma t)^{n}}{n !},
\end{array}
$$

where $w_{f} \approx \frac{W_{n}}{N_{n}}$ stands for the population of basis states of the class $n$ with $N_{n}$ as the number of states in this class (in fact, for $t \sim n \tau$ one needs to count only the states inside the energy shell since the population of the states outside the energy interval with $\left|E_{f}-E_{0}\right|>\Gamma$, is small, see Eq.(13)). Here we have used the relations $N_{n}=M^{n}$ and $\sum_{n=0}^{\infty} \frac{(\Gamma t)^{n}}{n !} n=\Gamma t \exp (\Gamma t)$. Two last terms in the right-hand-side of Eq.(21) turn out to be smaller than the first one, therefore, one can write,

$$
S(t) \approx \Gamma t \ln M[1+f(t)]
$$

with some function $f(t) \ll 1$ which slowly depends on time.

In this estimate for the increase of entropy, we did not take into account the influence of fluctuations of $w_{f}$. One can show that for gaussian fluctuations of the coefficients $A_{f}$ with the variance given by their meansquare values, for large number of principal components $N_{p c}(t) \equiv \exp (S(t))$ the entropy should be corrected by a small factor of the order of $\ln 2$ (see, for example, [30]).

If one neglects the second term in 222, we obtain a linear increase of the entropy, which means that the number of principal components $N_{p c}(t)$ increases exponentially fast with time. This behavior can be compared with a linear increase of dynamical entropy $S_{c l}(t)$ in classical chaotic systems where $S_{c l}(t)$ was found to be related to an exponential divergence of close trajectories in the phase space $\left(S_{c l}(t) \propto \lambda t\right.$ with $\lambda$ as the Lyapunov exponent, see, for example [31]). The non-trivial point is that the linear increase of entropy also occurs for systems without the classical limit, see recent paper [32].

Note, that for a small time the function $W_{0}(t)$ has the form $W_{0}(t)=\exp \left(-\Delta_{E}^{2} t^{2}\right)$ (see (9)), not the exponential dependence $\exp (-\Gamma t)$. Therefore, one should modify the expression for the entropy in order to make it valid for small times. For this, we replace $\Gamma t$ in Eq.(19) by a more accurate expression, $-\ln \left(W_{0}\right)$, which gives $\Delta_{E}^{2} t^{2}$ for small time $t \ll \Gamma / \Delta_{E}^{2}$ and $\Gamma t$ for large time $t \gg \Gamma / \Delta_{E}^{2}$ , therefore,

$$
W_{n}=\frac{\left(\ln W_{0}^{-1}\right)^{n}}{n !} W_{0} .
$$

It is easy to check that the normalization condition is fulfilled again,

$$
\sum_{n=1}^{\infty} W_{n}=\sum_{n=1}^{\infty} \frac{\left(\ln W_{0}^{-1}\right)^{n}}{n !} W_{0}=W_{0} \exp \left(\ln \left(W_{0}^{-1}\right)\right)=1 .
$$

For the entropy one obtains

$$
S=-\sum_{n=1}^{\infty} W_{n} \ln \left(\frac{W_{n}}{N_{n}}\right)
$$

At small time $t \ll \Gamma / \Delta_{E}^{2}$ the entropy is given by two terms, $n=0$ and $n=1$ (direct transitions), therefore,

$$
\begin{array}{r}
S(t)=-W_{0}(t) \ln W_{0}(t)-W_{1}(t) \ln \left(\frac{W_{1}}{N_{1}}\right) \\
\approx \Delta_{E}^{2} t^{2}\left(1+\ln \left(\frac{\Delta_{E}^{2} t^{2}}{N_{1}}\right)^{-1}\right)
\end{array}
$$

This expression can be compared with the direct calculation based on the relation $w_{f}=\left|H_{0 f} t\right|^{2}$,

$$
S(t)=\Delta_{E}^{2} t^{2}+t^{2} \sum_{f}\left|H_{0 f}\right|^{2} \ln \left(\frac{1}{H_{0 f}^{2} t^{2}}\right)
$$

There is an agreement between Eqs.(26) and (27) since $\Delta_{E}^{2}=\sum_{f}\left|H_{0 f}\right|^{2}=N_{1} \overline{H_{0 f}^{2}}$.

Let us now discuss the whole time dependence of the entropy, including large times $t$ when the system is close to the equilibrium. In a finite system of particles any basis state can be reached, starting from an initial state, in several "interaction steps" $\left(H_{0 \alpha} H_{\alpha \beta} H_{\beta \gamma} \ldots\right)$. For example, in the system of $n=6$ particles three steps is needed since the two-body interaction can not move more than two particles from one basis state to another. If the number of classes $n_{c}$ is finite, the states in the last class do not decay (there is no term $-\Gamma W_{n_{c}}$ in the last equation in (18)), and the probability to be in the last class is determined from the normalization condition $W_{n_{c}}=1-\sum_{k=0}^{n_{c}-1} W_{k}$. The additional condition is that the considered basis states should be inside the energy interval, $\left|E_{f}-E_{0}\right| \leq \min (\Gamma, \Delta E)$, thus limiting the number $N_{n}$ of the basis states in each class. Note that the value of $W_{n}$ is restricted from below by the equilibrium relation $W_{n} \geq N_{n} / N_{p c}$. These limitations make "exact" expression for the entropy very complicated. Instead, 
we can propose the following simple expression which is approximately valid in systems with a small number of classes $\left(n_{c} \sim 1\right)$,

$S(t)=-W_{0}(t) \ln W_{0}(t)-\left(1-W_{0}(t)\right) \ln \left(\frac{\left(1-W_{0}(t)\right)}{N_{p c}}\right)$

This expression takes into account the normalization condition $\sum_{f \neq 0} w_{f}=1-W_{0}$ and has a reasonable behavior for both small and large times.

\section{NUMERICAL RESULTS FOR THE ENTROPY}

Now we compare the obtained analytical expressions with numerical data for the TBRI model. For the case when the strength function (SF) has the Breit-Wigner form, the time dependence of the entropy is shown in Fig. 4 for the parameters of Fig. $2, n=6, m=12, V_{0}^{2} \approx$ $0.003, \Gamma_{0} \approx 0.50, \Delta_{E} \approx 1.16$, with the average over $N_{g}=2$ Hamiltonian matrices.

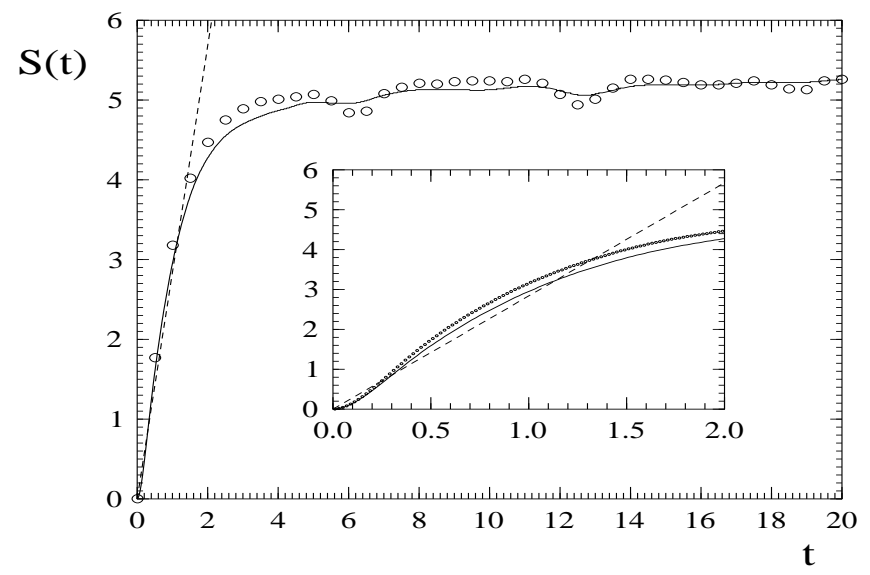

FIG. 4. Entropy versus time for the TBRI model in the case when the SF has the standard Breit-Wigner form. The parameters are the same as in Fig.2, with $N_{g}=2$. The circles stand for numerical data, the solid curve is the analytical expression (28), and the dashed line represents a linear slope according to an approximate expression (30). In the inset the same is shown for a smaller time scale.

The number $M$ of the basis states directly coupled by the random two-body interaction, is determined by the expression [7],

$$
M=n(m-n)+\frac{n(n-1)(m-n)(m-n-1)}{4},
$$

where the first term gives the number of one-particle transitions, and the second stands for two-particles transitions. In our case of $n=6$ particles and $m=12$ orbitals, the total number of basis states is $N=924$ and
$M=261$. The effective number of classes in the cascade model can be determined from the relation $M^{n_{c}}=N$. This gives $n_{c}=\ln N / \ln M \approx 1.2$. Thus, we can use the simple expression (28) to describe the dependence of the entropy on time analytically. The data in Figs.4-5 demonstrate an excellent agreement between the numerical and analytical results.

We should note that the theoretical dependence (28) which gives quite good approximate description of the data on the whole time scale, has the parameter $N_{p c}$ (effective number of principal components in the stationary distribution $w_{f}(t \rightarrow \infty)$ ) which is related to the limiting value of the entropy, $N_{p c}=\ln S(\infty)$. It can be estimated analytically as discussed above from the width of the energy shell; when plotting the solid curves in Figs.4-5 we have used the exact value found numerically.

To avoid confusion, we should explain that the actual number of classes in the case of $n=6$ particles and $m=12$ orbitals is equal to 3 since all basis states can be populated in the third order in the two-body interaction. However, the number of states in the second, $k=2$, and third, $k=3$, classes are much smaller than it may follow from the exponential relation $N_{k}=M^{k}$ (in practice, this relation may be justified for a large number of particles only). This is the reason why the one-class formula (28) works so well.

It is also instructive to compare the entropy with the linear time dependence,

$$
S(t)=\Gamma t \ln M
$$

that stems from Eq.(22) if the first term is taken only. This dependence globally corresponds to the data on some time scale, however, the actual dependence for $S(t)$ clearly differs from the linear one (see inset in Fig.4). Note that the quadratic increase of energy occurs on a very small time scale only. As for the oscillations of the entropy for a very large time close to an equilibrium, this phenomenon will be discussed below.
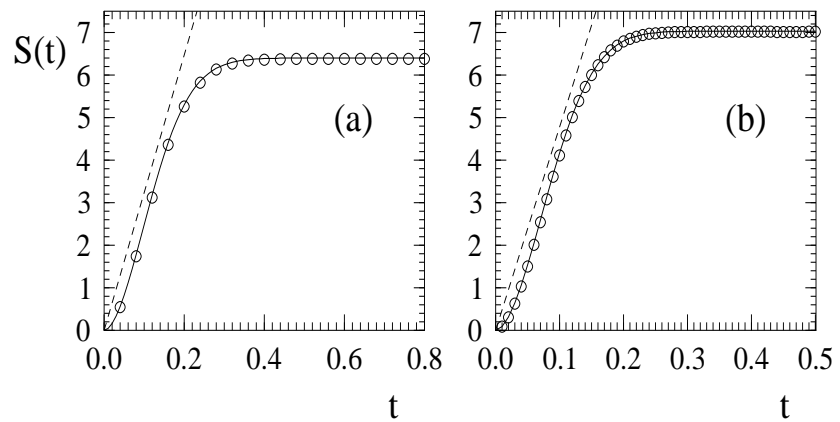

FIG. 5. Time dependence of the entropy for the TBRI model when the strength function is of the Gaussian form: (a) $n=6, m=12, V_{0} \approx 0.083, \Gamma_{0} \approx 10.5, \Delta_{E} \approx 5.8$, and (b) $n=7, m=13, V_{0} \approx 0.12, \Gamma_{0} \approx 14.6, \Delta_{E} \approx 8.13$. Circles are numerical data for $N_{g}=2$, solid curves stand for the approximate expression (28), and dashed lines represent the linear dependence (31). 
For a strong interaction, when the form of the SF is very close to the Gaussian, numerical data are reported in Fig. 5 for $n=6, m=12$ and for $n=7, m=13$. The interaction strength is chosen in order to have the same ratio $\Gamma_{0} / \Delta_{E} \approx 1.8$, as in Fig.4.

In this case the half-width of the strength function is determined by $\Delta_{E}$ since $\Gamma_{0}=2 \pi \rho_{f} H_{0 f}^{2}$ is larger than $\Delta_{E}$. As a result, the role of $\Gamma$ in the expressions (22) and (28) plays the width $\Delta_{E}$. In both cases numerical data give strong evidence of a linear increase of the entropy,

$$
S(t)=\Delta_{E} t \ln M
$$

before the saturation. It is clearly seen that this analytical estimate gives a correct value for the slope of $S(t)$. The shift is due to the initial time scale where the time dependence is quadratic, this fact is neglected in the estimate.

It should be pointed out that the linear dependence of $S(t)$ in Fig.5 is much more pronounced than in the BWregion (compare with Fig. 4 for which the SF is of the Breit-Wigner form). Our results indicate a clear difference between the two cases related to the Breit-Wigner and Gaussian forms of the SF. This point is somehow supported by recent studies [22,23 where it was shown that for a relatively weak interaction $\left(\Gamma_{0}\right.$ is small in comparison with $\Delta_{E}$ ) resulting in the Breit-Wigner form of the $\mathrm{SF}$, there is no detailed quantum-classical correspondence for the evolution of wave packets in the energy space. On the other hand, in the Gaussian region (with the Gaussian form for the SF), the detailed quantumclassical correspondence is possible 22,23. In the latter case one can expect a linear growth for the entropy, as it was found in classical models [31]. The principal difference between these two cases (in what concerns the quantum-classical correspondence and the possibility of the localization in the energy shell) for the first time has been discussed in Ref. [20].

\section{WIDTH OF PACKETS AND INVERSE PARTICIPATION RATIO}

The width of the wave function in the basis representation can be measured through the variance,

$$
\begin{gathered}
\Delta^{2}(t)=\sum_{f}\left(n_{f}-n_{0}\right)^{2}\left|A_{f}(t)\right|^{2}=\left(1-W_{0}(t)\right)\left|\Delta_{f}(t)\right|^{2} \\
\left|\Delta_{f}(t)\right|^{2}=\frac{\sum_{f}\left(n_{0}-n_{f}\right)^{2}\left|A_{f}(t)\right|^{2}}{\sum_{f}\left|A_{f}(t)\right|^{2}}
\end{gathered}
$$

where $n_{f}$ and $n_{0}$ label corresponding basis states, $f \neq 0$, and we have used the normalization condition $\sum_{f}\left|A_{f}(t)\right|^{2}=1-W_{0}(t)$. The function $\left|\Delta_{f}(t)\right|^{2}$ is a slow function of time, it changes from the effective bandwidth of the Hamiltonian matrix, entirely determined by the matrix elements $H_{0 f}$, to the final width of the wave packet in the basis representation, which is defined by the width of the energy shell (approximately equal to $\sqrt{2} \Delta E)$. Therefore, the leading time dependence is given by the term $1-W_{0}(t)$.

For relatively small times before the saturation, we can use the simple estimate $w_{f} \approx\left|H_{0 f} t\right|^{2}$, which results in the following quadratic dependence,

$$
\Delta^{2}(t) \approx t^{2} \sum_{f}\left(n_{f}-n_{0}\right)^{2} H_{0 f}^{2}=t^{2} V_{0}^{2} \Delta_{0}^{2}
$$

Here $\Delta_{0}$ is some constant related to an effective bandwidth of the Hamiltonian matrix. The linear dependence for the width of packet, $\Delta(t)=t V_{0} \Delta_{0}$, corresponds to a generic ballistic-like behavior of wave packets found for the WBRM model 22.

Note that the band-width of the Hamiltonian matrix can be much larger than the final width of the wave packet due to the dependence of the latter on the interaction strength. Therefore, the linear increase of $\Delta(t)$ can be very fast, and quickly it becomes saturated on the time scale of the applicability of the expansion in $H t$, see Eq. (33).

Before comparing the obtained expressions with numerical data, let us first analyze the time dependence of the number of principal components $N_{p c}(t)$ for wave packets in the basis representation. It is natural to define $N_{p c}$ through the entropy, $N_{p c}(t)=\exp (S(t))$. This definition has been widely used in different applications, see for example, 30.

The number of principal components can be also defined through the inverse participation ratio $l_{i p r}$ :

$$
\left(l_{i p r}\right)^{-1}=\sum\left|A_{f}\right|^{4} \approx \sum_{k} \frac{W_{k}^{2}}{N_{k}} \approx W_{0}^{2} \sum_{k} \frac{\left(\ln W_{0}^{-1}\right)^{2 k}}{k ! k ! N_{k}}
$$

Here we used Eq.(23) for $W_{k}$. The sum in Eq.(34) gives the following result for the infinite number of classes (this may be a reasonable approximation for time $\left.t<<n_{c} \tau\right)$

$$
\left(l_{i p r}(t)\right)^{-1}=W_{0}^{2} I_{0}\left(\frac{2 \ln \left(W_{0}^{-1}\right)}{\sqrt{M}}\right)
$$

where $I_{0}(z)$ stands for the modified Bessel function and we used the relation $N_{n}=M^{n}$.

This expression has the following asymptotics,

$$
\begin{aligned}
\left(l_{i p r}(t)\right)^{-1} & =W_{0}^{2}\left(1+\frac{1}{N_{1}}\left(\ln W_{0}^{-1}\right)^{2}\right) \\
& \approx 1-2 t^{2}(\Delta E)^{2}\left(1+\frac{1}{N_{1}}\right)
\end{aligned}
$$

for small time, and 


$$
\left(l_{i p r}(t)\right)^{-1}=\exp \left(-2 \Gamma\left(1-\frac{1}{\sqrt{M}}\right) t\right)
$$

for large time. Therefore, $N_{p c}$ defined through the inverse participation ratio $l_{i p r}$ also may have an interval of exponential increase in time (if the number of classes $n_{c}$ is not small and we can extend the summation over $k$ in Eq. (34) to infinity). Here we again neglected the fluctuations of $A_{f}(t)$ which may increase the value of $l_{i p r}^{-1}$ up to the factor 3 .

For a system with small number of classes on can suggest the following approximate expression,

$$
\left(l_{i p r}(t)\right)^{-1}=W_{0}^{2}+\frac{\left(1-W_{0}\right)^{2}}{l_{i p r}(\infty)} .
$$

This expression takes into account the normalization condition $\sum_{f \neq 0} w_{f}=1-W_{0}$ and has a reasonable behavior for both small and large time.

In any system with finite number of particles the energy shell contains finite number of basis states. For a stationary chaotic state the number of principal components is estimated as $N_{p c}^{s t} \sim \Gamma / D$ where $D$ is the mean energy distance between many-particle levels (we assume here that the spreading width $\Gamma$ of exact eigenstates is less than $\Delta_{E}$ ). In the non-stationary problem this leads to the saturation of $N_{p c}(t)$ to the value $N_{p c}(\infty) \simeq 2 N_{p c}^{s t}$, and to the maximal value $S \approx \ln N_{p c}(\infty)$ for the entropy (see above and in Ref. [17]).

Numerical data for the TBRI model for the case when the $\mathrm{SF}$ is of the Breit-Wigner form are summarized in Fig.6 for the same parameters as in Fig.2 and Fig.4. Three quantities are plotted here: the width $\Delta(t)$, the number of principal components $N_{p c}(t)=\exp (S(t))$ and $l_{i p r}(t)=\left(\sum\left|A_{f}(t)\right|^{4}\right)^{-1}$ determined by the inverse participation ratio. For $N_{p c}(t)$ two curves are given, one is due to the analytical expression (28) for the entropy, and another is computed directly from the evolution of the TBRI model, with additional average over $N_{g}=2$ number of realization of random Hamiltonian.

From the reported data one can see that the time dependence of the width $\Delta(t)$ of packets is quite simple. Namely, on the first very short time scale the increase of the width is linear in time (see also inset in Fig.6 where $\Delta(t)$ is shown on this time scale), and after, the width quickly saturates. This behavior is in the correspondence with analytical estimates discussed above, and with numerical results found in the WBRM model [22].

In contrast to this time dependence, the increase of the number of principal components $N_{p c}(t)$ is very different, both for $N_{p c}(t)$ defined by the entropy and for the $l_{i p r}(t)$ determined by the inverse participation ratio. Indeed, both $N_{p c}(t)$ and $l_{i p r}(t)$ increase slowly in time before the saturation to their limit values. The absolute difference between these two quantities is not important since the definition of $l_{i p r}$ is given up to some factor which is sensitive to the type of fluctuations in $A_{f}(t)$. As we already noted, the gaussian fluctuations decrease the value of $l_{i p r}$ by the factor 3 .

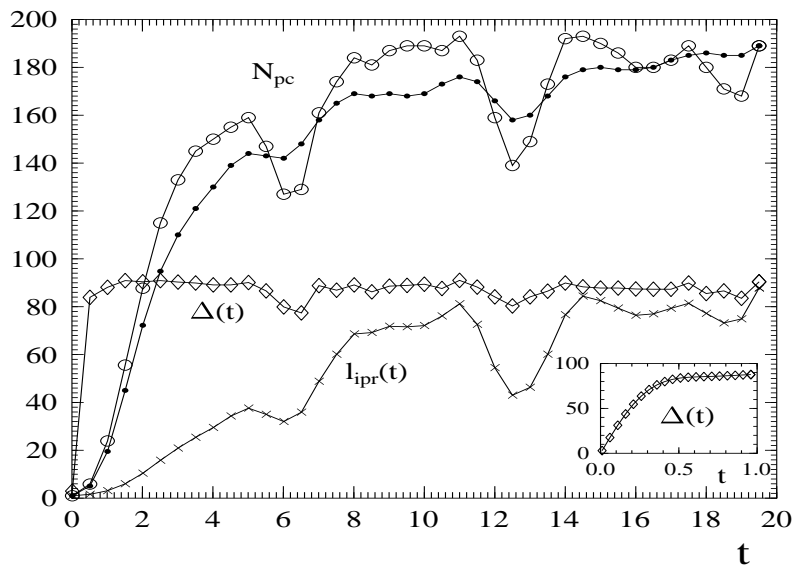

FIG. 6. Time dependence of different quantities for the TBRI model in the case of the BW-form of strength functions. The parameters are the same as in Fig.4, with $N_{g}=2$. Circles stand for numerical data for $N_{p c}=\exp (S(t))$ with $S(t)$ taken from Fig.4, dots correspond to the analytical expression for $N_{p c}(t)$ with $S(t)$ from Eq. 28), triangles represent numerical data for the width $\Delta(t)$, and squares are numerical results for $l_{\text {ipr }}(t)$. The width $\Delta(t)$ on a smaller time scale is shown in the inset.

One of the most interesting facts which can be drawn from these data is a big difference for characteristic time scales which correspond to the saturation. Indeed, if the width $\Delta(t)$ completely saturates at time $t \approx 0.5$, both $N_{p c}$ and $l_{i p r}$ manifest a very slow saturation by the time $t \approx 20$. This means that the mechanism for the width increase is different from that responsible for the increase of the number of principal components in the wave packet.

To explain this phenomenon let us consider the initial time scale for the time dependence of $N_{p c}$ and $l_{i p r}$, where one can detect an approximate linear increase of the entropy $S(t)$. The very point is that at small times $t \leq \tau$ the wave function has a large number of holes since only directly connected basis states are populated. For a system with a large number $n$ of particles the fraction of these states is exponentially small $(\sim \exp (-n))$ due a two-body nature of the interaction. With an increase of time, for $t \geq \tau=\Gamma_{0}^{-1}$, the states in other classes start to be filled and the holes begin disappear. This stage for $t<n_{c} \tau$ corresponds to an exponential increase of the number of principal components and linear increase of the entropy.

It is instructive to analyze the evolution of wave packets on a smaller time scale of the ballistic spread, see Fig.7. These data confirm theoretical expectations according to which at small times only those basis states that belong to the first class, are involved in the dynamics. Indeed, large gaps are clearly seen in the distribution 
of $w_{f}$, which persist during all the time of the ballisticlike spread. These gaps reduce the number of the principal components, however, they are not important for the calculation of $\Delta(t)$, see Eq.(32).

An important peculiarity of the wave dynamics is that initially all basis states of the first class are excited. One can see that for a very small time $t=0.2$ the whole available region $1<f<N$ is filled with approximately the same amplitudes $w_{f}=\left|H_{0 f} t\right|^{2}$. With an increase of time, the amplitudes grow and form the envelope of the packet, in accordance with Eq. 13). One should stress that the quadratic time dependence for the second moment of a packet on this short-time scale occurs not due to a linear spread of the front of the wave packet, but due to a specific growth of the amplitudes of those basis states which are located inside the energy shell.
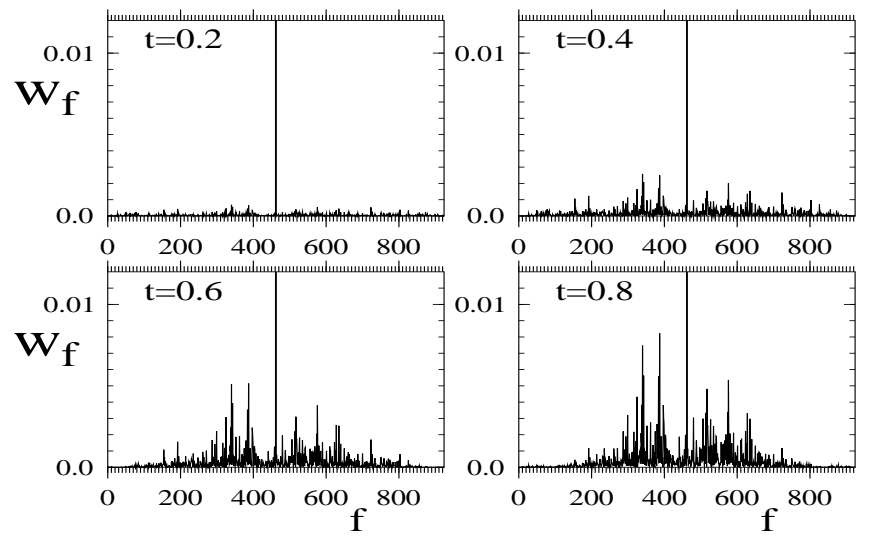

FIG. 7. Wave packet $w_{f}(t)$ for the TBRI model at different times $t=0.2,0.4,0.6 .0 .8$ for the parameters of Fig.7. One particular Hamiltonian matrix is used without any additional average.

The remarkable effect is a kind of oscillations for all quantities of Fig.6. Similar oscillations (however, not so strong) are also present in the time dependence of the entropy $S(t)$, see Fig.4. Since the period $T \approx 6.5$ of these oscillations is much larger than the time scale $t \approx 0.5$ of the ballistic spread of wave packets, it is clear that this effect is entirely related to the dynamics in the Fock space formed by different classes. The origin of these oscillations can be explained in terms of the cascade model discussed in Section III. Indeed, one can expect a strong effect of a reflection due to the finiteness of the Fock space. The first reflection occurs for $t_{0} \approx n_{c}\left(\Gamma_{0}\right)^{-1}$, therefore, the period of oscillation is $T \approx 2 t_{0}$. One can see that this estimate gives the correct result for $T$ with $n_{c} \approx 1.5$. The latter value is close to our rough estimate $n_{c} \sim 1.2$ for an effective number of classes.

To compare the data for $l_{i p r}$ defined by the inverse participation ratio with the analytical expression (38), we have plotted separately both results for a larger time scale $t \leq 40$ in Fig.8. One can see that our estimate (38) gives quite accurate description of the data on a large time scale up to $t \approx 20$. After this time, the saturation occurs and all local time dependence may be treated as fluctuations around the limiting value. The data presented in this figure give strong evidence of the effectiveness of our analytical approach.

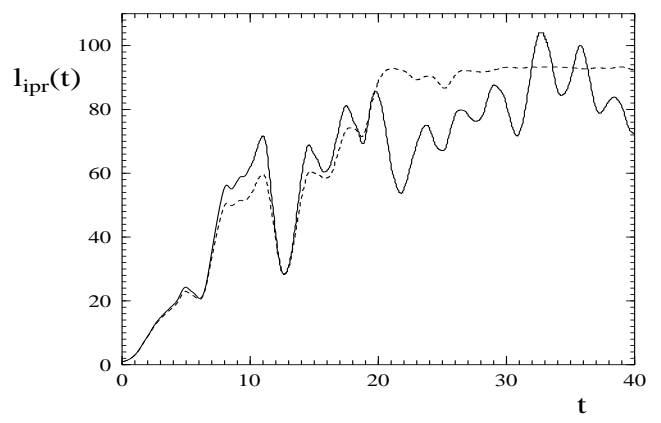

FIG. 8. Time dependence of $l_{i p r}(t)$ for the parameters of Fig.6 on a larger time scale for one Hamiltonian matrix. Solid curve stand for numerical data, and the dashed curve corresponds to the analytical expression (38).

Let us now come to the case when the form of the strength function is close to the Gaussian. In Fig.9 one can see that the analytical expressions connecting the probability $W_{0}(t)$ to stay in an initial basis state, with the time dependence of the number of principal components $N_{p c}(t)=\exp (S(t))$ and $l_{i p r}(t)$, give correct global description of numerical data. When comparing with the previous case of the BW-form of the SF, see Figs.6,8, one can conclude that for a strong interaction (when the Gaussian dependence for the SF emerges) the behavior of all quantities do not reveal strong oscillations. This effect is related to the fact that the time scale for the saturation of the width of packets is of the same order as for the saturation of both $N_{p c}$ and $l_{i p r}$. In such a case the effect of reflection in the Fock space is suppressed by a strong spread of packets in the energy shell.

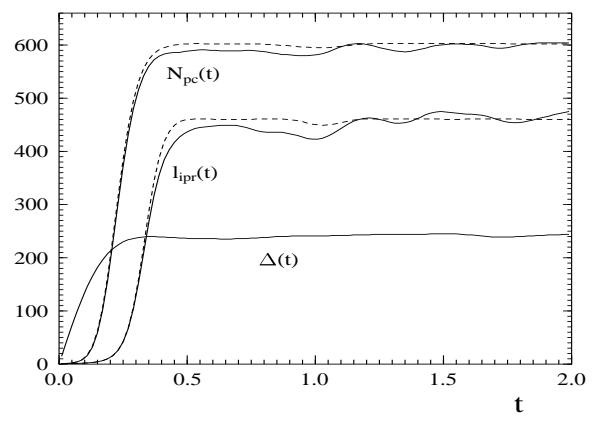

FIG. 9. The same quantities as in Fig.6 for a strong interaction with the Gaussian form of the SF. The parameters for the TBRI model are the same as in Fig.5a: $n=6, m=12, V_{0} \approx 0.083, \Gamma_{0} \approx 10.5, \Delta_{E} \approx 5.8$. Solid curves correspond to numerical data, dashed curve stand for the corresponding analytical expressions: Eq.(38) for $l_{i p r}(t)$, and Eq. 28) for $S(t)$ in the definition $N_{p c}(t)=\exp (S(t))$. 
One can see, that there is a quite strong difference in the time-dependence of all discussed above quantities for the two extreme cases of the Breit-Wigner and Gaussian forms of the strength function. In the BW-case the two effects (ballistic-like spread of packets and the cascadelike evolution in the Fock space) have very different time scales, and both these effects can be distinguished in the dynamics. Contrary, in the second case of the Gaussian form of the SF, two time scales are comparable. Therefore, the two effects coexist on the same time scale and, as a result, the global time-dependence turns out to be much simpler.

\section{COMPARISON WITH WIGNER BAND RANDOM MATRICES}

In this Section we discuss numerical results obtained for the WBRM model for the same quantities as the considered above. The dynamics of wave packets in this model has been recently studied in 22] in the connection with the problem of quantum-classical correspondence. Here, instead, we concentrate our attention on the correspondence between the evolution of packets in the WBRM and TBRI models.

As was mentioned, the WBRM model is quite close to the TBRI model. It consists of two parts, one of which is a diagonal matrix with increasing "energies" $\epsilon_{j}$ and another is a band matrix $V_{i j}$,

$$
H_{i j}=\epsilon_{j} \delta_{i j}+V_{i j},
$$

where $\delta_{i j}$ is the delta-function. In original papers 19 the "unperturbed spectrum" was taken in the form of the "picked fence", $\epsilon_{j}=j D$, where $D=\rho_{0}^{-1}$ is the spacing between two close energies and $j$ is a running integer number. We consider here the case with random values $\epsilon_{j}$ with the mean spacing $D$, reordered in an increasing way, $\epsilon_{j+1}>\epsilon_{j}$. As for the off-diagonal matrix elements $V_{i j}$, they are assumed to be gaussian random and independent variables inside the band $|i-j| \leq b$, with the zero mean, $\left\langle V_{i j}=0\right\rangle$, and the variance $\left\langle V_{i j}^{2}\right\rangle=V_{0}^{2}$. Outside the band, the matrix elements vanish. Thus, the control parameters of this model are the ratio $V_{0} / D$ of a typical matrix element to the mean level spacing, and the band-width $b$. As one can see, the first term in (39) corresponds to a "mean field" $H_{0}$, and the interaction $V$ has a finite energy range.

For the first time the SF for the WBRM has been analyzed in Ref. [19]. It was analytically found that the form of the strength function essentially depends on one parameter $q=\frac{\rho_{0}^{2} V_{0}^{2}}{b}$ only. Wigner has proved [19] that for a relatively strong perturbation, $V_{0} \gg D$, in the limit $q \ll 1$ the form of the LDOS is the Lorentzian,

$$
W_{B W}(\tilde{E})=\frac{1}{2 \pi} \frac{\Gamma_{B W}}{\tilde{E}^{2}+\frac{1}{4} \Gamma_{B W}^{2}}, \quad \tilde{E}=E-D j,
$$

which nowadays is known as the Breit-Wigner (BW) dependence. Here the energy $\tilde{E}$ refers to the center of the distribution. The half-width $\Gamma_{B W}$ of the distribution (40) is given by the Fermi golden rule,

$$
\Gamma_{B W}=2 \pi \rho_{0} V_{0}^{2}
$$

In other limit $q \gg 1$ the influence of the unperturbed part $H_{0}$ can be neglected and the shape of the SF tends to the shape of density of the states of the band random matrix $V$, which is known to be the semicircle.

Recently, Wigner's results have been extended in 21] to matrices $H$ with a general form of $V$, when the variance of the off-diagonal matrix elements decreases smoothly with the distance $r=|i-j|$ from the principal diagonal. In this case the effective band size $b$ is defined by the second moment of the envelope function $f(r)$. Another important generalization of the WBRM studied in [21], is an additional sparsity of the matrix $V$, which may mimic realistic Hamiltonians. In such a form, the WBRM model is closer to the TBRI model, however, in the latter the sparsity of the interaction is due to a two-body nature of the interaction. As a result, the positions of zero elements are not completely random as in the WBRM model, see details in [7,9].

Random matrix models of the type (39) are very useful for the understanding generic properties of the SF. The condition for the SF to be of the BW form in the WBRM model has simple form [21],

$$
D \ll \Gamma_{B W} \ll \Delta_{b} ; \quad \Delta_{b}=b D
$$

The left part of this relation indicates the nonperturbative character of an interaction, according to which many of unperturbed basis states are strongly coupled by an interaction. On the other hand, the interaction should not be very strong, namely, the width $\Gamma_{B W}$ determined by Eq.(41), has to be less than the width $\Delta_{b}$ of the interaction in the energy representation. The latter condition is generic for systems with finite range of the interaction $V$. One should stress that, strictly speaking, the BW form (40) is not correct since its second moment diverges. As was shown in [19,1, outside the energy range $|\tilde{E}|>\Delta_{b}$ the SF in the model (39) decreases with the energy faster than a pure exponent.

Note, that in the TBRI model the energy scale $\Delta_{b}$ is irrelevant since there is no sharp border of the interaction and $\Delta_{b}$ is of the order of the whole energy spectrum. For this reason, instead of $\Delta_{b}$ it is more convenient to use the variance $\Delta_{E}^{2}$ of the SF which may have the classical limit [20,22]. The latter quantity can be expressed through the off-diagonal matrix elements of the interaction, $\Delta_{E}^{2}=\sum_{j} V_{i j}^{2}$ for $i \neq j$, therefore, $\Delta_{E}^{2}=2 b V_{0}^{2}$. As a result, we have $\Delta_{b}=\pi \Delta_{E}^{2} / \Gamma_{B W}$ and Eq.(42) can be written as 


$$
D \ll \Gamma_{B W} \ll \Delta_{E} \sqrt{\pi} .
$$

Numerical data 27] for the WBRM model show that on the border $\Gamma_{B W} \approx 2 \Delta_{E}$ the form of the SF is quite close to the Gaussian, and this transition from the BW dependence to the Gaussian-like turns out to be quite sharp. Although the extreme limit of a very strong interaction, $q \gg 1$, (or, the same, $\Gamma_{B W} \gg \Delta_{E}$ ) has been studied by Wigner in the WBRM model (39), the semicircle form of the SF seems to be unphysical. Indeed, this form is originated from the semicircle dependence of the total density $\rho_{V}(E)$ defined by $V$ only, therefore, when neglecting the term $H_{0}$. Thus, the case $V \gg H_{0}$ in terms of the TBRI model means that the residual interaction is much stronger than the mean-field part $H_{0}$, which is physically irrelevant.

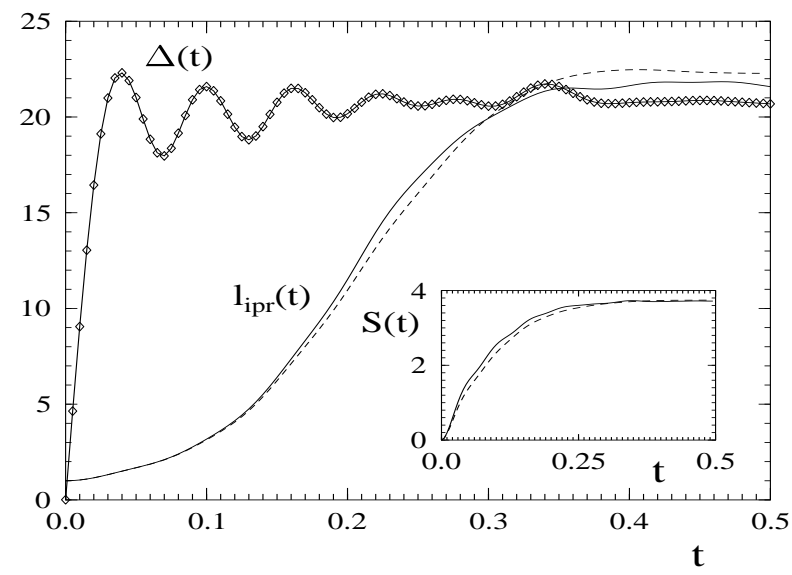

FIG. 10. Time dependence of the entropy $S(t)$ (in the inset), the width of the packet $\Delta(t)$ and the number of principal components $l_{i p r}(t)$ for the WBRM model for the case of the BW-form of the SF. The parameters are: $N=924, b=110, D=1.0, V_{0}=1.0$, correspondingly, $\Gamma_{B W} \approx 6.28, \Delta_{E} \approx 14.8$. Solid curves for $S(t)$ and $l_{i p r}(t)$ are numerical data, dashed curves represent analytical expressions (28) (for $S(t)$ ) and (38) (for $l_{i p r}$ ). Diamonds, connected by the solid line, correspond to numerical data for $\Delta(t)$.

Numerical data for the WBRM model in the case of the BW-dependence of the SF are given in Fig.10. When comparing with the corresponding quantities discussed above for the TBRI model (see Fig.6) we should note the following. First of all, one can see that the simple analytical expression (28) gives a correct description of the increase and saturation of the entropy $S(t)$. The same we can say about the expression (38) for the number of principal components defined through the inverse participation ratio.

Second, we would like to stress that the global time dependence for all quantities is quite similar to that found for the one-class variant of the TBRI model. Relatively simple structure of the Wigner band random matrices allows one to perform a detailed comparison of the data with analytical estimates. Indeed, the application of the relation (33) for the WBRM model gives,

$$
\Delta^{2}(t)=\frac{2}{3} t^{2} V_{0}^{2} b^{3},
$$

see also Ref. [18]. Therefore, for the parameters of Fig.10 we have $\Delta(t)=B t$ with $B=\sqrt{\frac{2}{3}} b^{3 / 2} V_{0} \approx 950$ which is in a good agreement with numerical data. We can also find the critical time $t_{d}$ after which the ballistic spread of the packet terminates. For this, we estimate the maximal width $\Delta_{m}$ of packet via the width $\Delta_{E}$ of the SF, $\Delta_{m} \approx \sqrt{2} \Delta_{E}$. This leads to the estimate $t_{m} \approx \sqrt{6} / b$, therefore, for Fig. 3 we have $t_{m} \approx 21$ which perfectly corresponds to the data. The latter estimates have been also checked for other values of $V_{0}$ and $b$, with the same good correspondence between simple estimates and numerical data.

Comparing global time dependence of the quantities presented in Fig.10 with the results for the TBRI model, see Fig.6, one can see that the main difference is the type of oscillations for the width of packets $\Delta(t)$. Namely, in contrast to the TBRI model where the period of oscillations is much larger than the time scale $t_{m}$ of the ballistic spread, in Fig.10 the period $T$ is just defined by the ballistic spread, $T \approx 2 t_{m}$. This very fact demonstrate the principal difference between the two models.

Indeed, for the WBRM model there is no specific evolution in the Fock space which is due to a two-body nature of interaction. Formally, the cascade model can be applied to the WBRM model with the number of classes $n_{c}=1$, since all states within the energy band $\Delta_{b}=b D$ start to be involved in the dynamics immediately. This means that in contrast to the TBRI model, in the WBRM model there is only one mechanism for the oscillations, namely, the reflection inside the energy shell which is populated ergodically, when time is running. No oscillations are detected for the number of principal components (the data for larger times are not shown), this confirms our conclusion about one kind of the reflection from the edges of the energy shell. It is interesting to note that the number of principal components does not reveal noticeable oscillations on the time scale of the ballistic spread since on this scale the value of $N_{p c}$ is very small.

The difference between these two models can be also seen when comparing the structure of wave packets in the basis representation at some instant times before the saturation (compare Fig.11 with Fig.7). In contrast to Fig.7 where many "holes" can be realized in the distribution $w_{f}$, for the WBRM model the filling of the available energy range of size $2 b D$ occurs ergodically. In both cases very strong fluctuations are present, which are expected to be gaussian, see discussion in [8]. It is important to stress, that in order to reveal this difference, 
we should avoid the ensemble average which washes out the presence of holes (if different Hamiltonian matrices have different unperturbed spectrum). This fact reflects one of basic peculiarities of the TBRI model, namely, the non-ergodic character of the matrices (the average over the spectrum inside one (very big) matrix may give completely different result from that obtained by an ensemble average, see references in the review [29]).
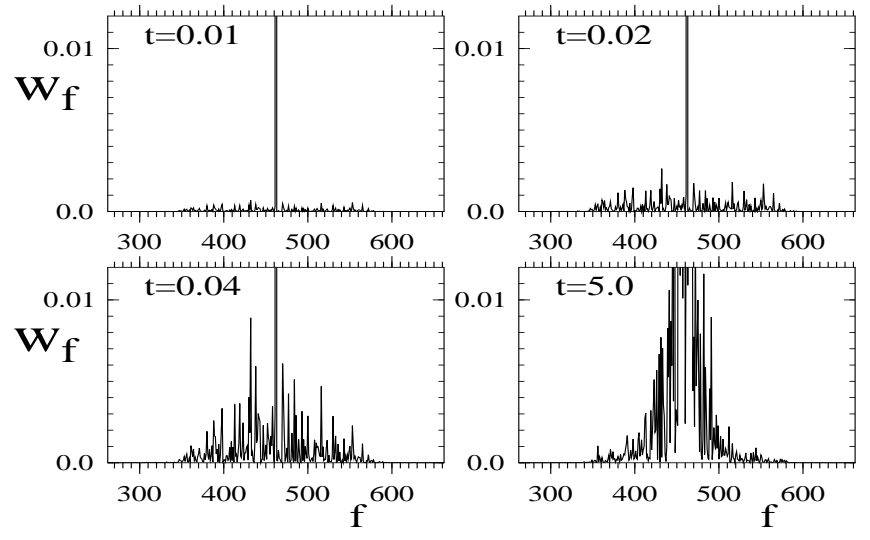

FIG. 11. Wave packet $W_{n}(t)$ for the WBRM model at different times $t=0.01,0.02,0.04,5.0$ for the parameters of Fig.10. One particular band random matrix is used without any additional average.

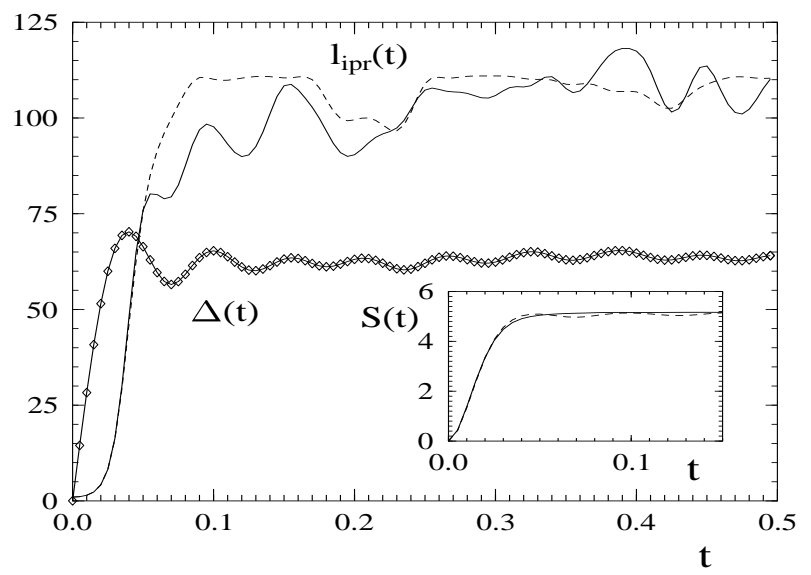

FIG. 12. The same as in Fig.10 for the case when the form of the $\mathrm{SF}$ is close to the Gaussian. The parameters are: $N=924, b=110, D=1.0, V_{0}=3.0$, correspondingly, $\Gamma_{B W} \approx 56.5, \Delta_{E} \approx 45.5$.

Finally, we present the data for the WBRM in the case of the Gaussian form of the strength function, see Fig.12. Here we also can see oscillations for the width of packets. As for the number of principal components found from the inverse participation ratio, numerical data manifest the oscillations, as well, with the same period as for the width of packets. In average, the numerical data for $S(t)$ and $l_{i p r}$ are well described by the simple analytical expressions relating these quantities to the probability $W_{0}(t)$ to stay in the initial state.

The most interesting result which can be drawn from the numerical data presented in Fig.10 and Fig.12, is a clear difference in the time dependence of the entropy $S(t)$. Comparing the data in the insets, one can conclude that linear increase of the entropy occurs for the case when the form of the SF is close to the Gaussian, but not for the case of the BW-form. Indeed, it is hard to indicate clear time scale of a linear increase of $S(t)$ in Fig.10, in contrast to Fig.12 where the linear time dependence is clearly seen (apart from a very small time scale). This very fact may be of quite generic, since in the TBRI model we also see a non-linear character of the entropy increase in the BW-regime.

\section{ACKNOWLEDGEMENTS}

This work was supported by the Australian Research Council. One of us (F.M.I.) gratefully acknowledges the support by CONACyT (Mexico) Grant No. 34668-E.

[1] V. V. Flambaum, A. A. Gribakina, G. F. Gribakin, and M. G. Kozlov, Phys. Rev. A 50, 267 (1994).

[2] G.F. Gribakin, A.A. Gribakina, V.V. Flambaum. Aust.J.Phys. 52, 443 (1999).

[3] M.Horoi, V.Zelevinsky and B.A.Brown, Phys. Rev. Lett. 74, 5194 (1995); V.Zelevinsky, M.Horoi and B.A.Brown, Phys. Lett. B 350, 141 (1995); N.Frazier, B.A.Brown and V.Zelevinsky, Phys. Rev. C 54, 1665 (1996); V.Zelevinsky, B.A.Brown, M. Horoi and N.Frazier, Phys. Rep., 276 , 85 (1996).

[4] V.V. Flambaum, Proc. 85th Nobel Symposium, Phys. Scr. 46, 198 (1993).

[5] B. Georgeot and D.L. Shepelyansky, Phys. Rev. Lett. 81, 5129 (1998).

[6] J. B. French and S. S. M. Wong, Phys. Lett. B 35, 5 (1970); O. Bohigas and J. Flores, Phys. Lett. B 34, 261 (1971).

[7] V.V. Flambaum, G.F. Gribakin and F.M. Izrailev, Phys. Rev. E 53, 5729 (1996).

[8] V.V.Flambaum and F.M.Izrailev, Phys. Rev. E 56, 5144 (1997).

[9] F.M.Izrailev, in Proceedings of the International School of Physics "Enrico Fermi", Course CXLIII, Varenna 2030 July, eds. G.Casati, I.Guarneri and U.Smilansky, IOS Press, 2000, pp.371-430. 
[10] T. Guhr, A. Müller-Groeling, and H.A. Weidenmüller, Phys. Rep., 200, 189 (1999).

[11] B.L. Altshuler, Y. Gefen, A. Kamenev and L.S. Levitov, Phys. Rev. Lett., 78, 2803 (1997).

[12] S. Äberg, Phys. Rev. Lett., 26, 3119 (1990); V.V.Flambaum, F.M.Izrailev, and G.Casati, Phys. Rev. E 54, 2136 (1996); V.V. Flambaum and F.M.Izrailev, Phys. Rev. E 55, R13 (1997); D.L. Shepelyansky and O.P. Sushkov, Europhys. Lett. 37, 121 (1997); B. Georgeot and D.L. Shepelyansky, Phys. Rev. Lett. 794365 (1997); P.Jacquod and D.L.Shepelyansky, Phys. Rev. Lett. 79, 1837 (1997); P.Jacquod and D.L.Shepelyansky, Phys. Rev. Lett. 79, 1837 (1997); Mejia-Monasterio, J.Richert, T.Rupp and H.A.Weidenmüller, Phys. Rev. Lett., 81, 5189 (1998); P.G. Silvestrov, Phys. Rev. Lett.,79, 3994 (1997); Phys. Rev. E. 58, 5629 (1998);

[13] V.V.Flambaum and F.M.Izrailev, Phys. Rev. E., 61, 2539 (2000).

[14] V.V. Flambaum, O.P. Sushkov, Nucl. Phys. A 412, 13 (1984). V.V. Flambaum, G.F. Gribakin. Progress in particle and nuclear physics, 35, Ch. 5, 423 (1995).

[15] G.E. Mitchell, J.D. Bowman and H.A. Weidenmüller, Rev. Mod. Phys. 71 , 445 (1999)

[16] B. Georgeot and D.L. Shepelyansky, quant-ph/9909074; quant-ph/0005015.

[17] V.V. Flambaum. Aust.J.Phys. 53, 489 (2000); quant$\mathrm{ph} / 9911061$.

[18] F.M. Izrailev, T. Kottos, A. Politi, S. Ruffo and G. Tsironis, Phys. Rev. E., 55, 4951 (1997).

[19] E.P. Wigner, Ann. Math. 62, 548 (1955); Ann. Math. 65,
203 (1957).

[20] G. Casati, B.V. Chirikov, I. Guarneri and F.M. Izrailev, Phys. Lett. A 223 (1996) 430-435.

[21] Y.V. Fyodorov, O.A. Chubikalo, F.M. Izrailev and G. Casati, Phys. Rev. Lett., 76, 1603 (1996).

[22] D. Cohen, F.M.Izrailev, and T.Kottos, Phys. Rev. Lett., 84, 2052 (2000).

[23] D. Cohen and T. Kottos, nlin.CD/0001026; condmat/0004022.

[24] F.M. Izrailev, to be published in the Proceedings of the Nobel Simposia "Quantum Chaos Y2K", Physica Scripta, 2001; cond-mat/0009207.

[25] A. Bohr and B. Mottelson, Nuclear structure, Vol. 1 (Benjamin, New York, 1969).

[26] V.V. Flambaum and F.M. Izrailev, submitted to Phys. Rev. E; quant-ph/0102088.

[27] G. Casati, V.V. Flambaum, and F.M. Izrailev, to be published.

[28] V.K.B. Kota and R. Sahu, nucl-th/0006079.

[29] T.A. Brody, J. Flores, J.B. French, P.A. Mello, A. Pandey, and S.S.M. Wong, Rev. Mod. Phys. 53, 385 (1981).

[30] F.M. Izrailev, Phys.Rep. 196, 299 (1990).

[31] V. Latora and M. Baranger, Phys. Rev. Lett., 82, 520 (1999); A.K. Pattanayak, Phys. Rev. Lett., 83, 4526 (1999); M. Barranger, V. Latora, and A. Rapisarda, cond-mat/0007302.

[32] P. Grigolini, M.G. Pala, and L. Palatella, condmat/0007323 